\title{
Disc structure of certain chamber graphs
}

\author{
P. J. Rowley \\ Abstract
}

The discs of chamber graphs for group geometries, including certain minimal parabolic geometries, maximal $p$-local geometries, Petersen geometries, GABs and Buekenhout geometries, are investigated.

Keywords : chamber graphs, group geometries, minimal parabolic geometries, maximal $p$-local geometries, Buekenhout geometries

MSC 2000: 05E20

\section{Introduction}

A chamber system over the set $I$ consists of a set $\mathcal{C}$ and a system $\left(\mathcal{P}_{i}\right)_{i \in I}$ of partitions of $\mathcal{C}$ indexed by $I$. The elements of $\mathcal{C}$ are called chambers and, for brevity, the system $\left(\mathcal{C},\left(\mathcal{P}_{i}\right)_{i \in I}\right)$ will often be referred to as the chamber system $\mathcal{C}$. Two chambers which are both in the same member of $\mathcal{P}_{j}$ for some $j \in I$ are said to be adjacent chambers. The chamber graph of the chamber system $\mathcal{C}$ is the graph with vertex set $\mathcal{C}$ and two (distinct) chambers are adjacent (in the chamber graph) if they are adjacent chambers in $\mathcal{C}$. (Note that a chamber is not adjacent to itself in the chamber graph.) If two chambers $c, c^{\prime}$ are both in the same member of $\mathcal{P}_{i}(i \in I)$, then we say they are $i$-adjacent, and denote this by $c \sim_{i} c^{\prime}$ (or $c^{\prime} \sim_{i} c$ ). The rank of the chamber system is the cardinality of $I$. An automorphism of $\mathcal{C}$ is a permutation $\sigma$ of $\mathcal{C}$ which preserves each of the partitions $\mathcal{P}_{i}$, that is, whenever $c \sim_{i} c^{\prime}\left(c, c^{\prime} \in \mathcal{C}, i \in I\right)$ then $c \sigma \sim_{i} c^{\prime} \sigma$.

We shall now concentrate on the following situation.

Hypothesis 1.1. $\mathcal{C}$ is a chamber system over $I$ and $G \leq \operatorname{Aut} \mathcal{C}$ is such that

(i) $G$ is transitive on $\mathcal{C}$; and

(ii) for each $i \in I, G$ is transitive on the members of the partition $\mathcal{P}_{i}$. 





\section{Disc structures}

\subsection{Group $G=\mathrm{L}_{2}(11)$}

GeOMetry: Petersen Geometry

$$
|G|=2^{2} \cdot 3 \cdot 5 \cdot 11=660
$$

page 5 / 27

go back

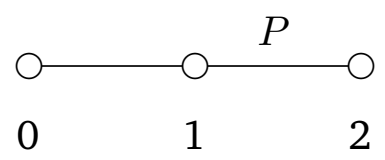

$G_{0} \cong \operatorname{Alt}(5), G_{1} \cong \operatorname{Dih}(12) \cong G_{2}$,

$G_{01} \cong \operatorname{Sym}(3), G_{02} \cong 2^{2} \cong G_{12}$,

$B \cong 2$ (see [8, p. 944]).

Number of Chambers: 330

DIAMETER: 9

\begin{tabular}{|c|c|c|c|c|c|c|c|c|c|}
\hline DISC & 1 & 2 & 3 & 4 & 5 & 6 & 7 & 8 & 9 \\
\hline SIZE & 4 & 8 & 15 & 26 & 42 & 58 & 76 & 68 & 32 \\
\hline
\end{tabular}

\subsection{Group $G=\hat{S}_{6}(\cong 3 \cdot \operatorname{Sym}(6))$}

GEOMETRY: 3-fold cover of the

$\mathrm{Sp}_{4}(2)$-quadrangle

$$
|G|=2^{4} \cdot 3^{3} \cdot 5=2,160
$$

Number OF ChAMBers: 135

DIAMETER: 8

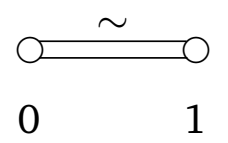

$G_{0} \cong 2^{3} \operatorname{Sym}(3) \cong G_{1}$,

$B \cong \operatorname{Dih}(8) \times 2$.

This geometry appears as a residue in the minimal parabolic geometries for $\mathrm{M}_{24}$, .1, M, He and $\mathrm{Fi}_{24}^{\prime}$ - see [16].

\begin{tabular}{|c|c|c|c|c|c|c|c|c|}
\hline DISC & 1 & 2 & 3 & 4 & 5 & 6 & 7 & 8 \\
\hline SIZE & 4 & 8 & 16 & 32 & 48 & 16 & 8 & 2 \\
\hline
\end{tabular}

\subsection{Group $G=\operatorname{Alt}(7)$}

$$
|G|=2^{3} \cdot 3^{2} \cdot 5 \cdot 7=2,520
$$


1. GEOMETRY:

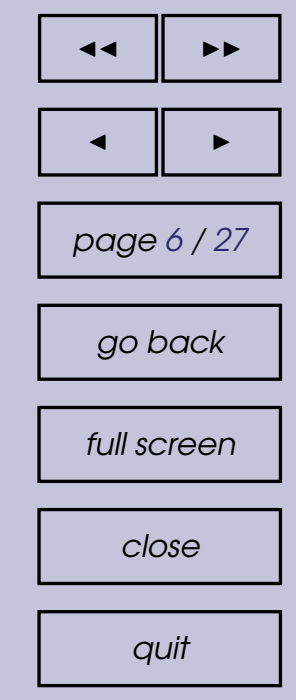



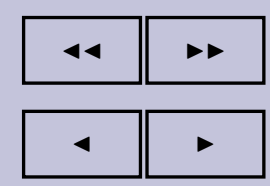

page $7 / 27$

go back

full screen

close

quit

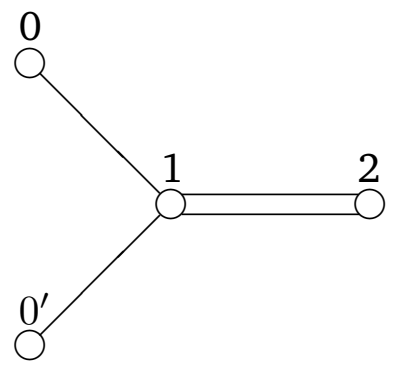

(See [14, p. 54].)

$B \cong \operatorname{Dih}(8)$,

$P_{i} \cong \operatorname{Sym}(4)$ for $i \in\left\{0,0^{\prime}, 1,2\right\}$.

\begin{tabular}{|c|c|c|c|c|c|}
\hline DISC & 1 & 2 & 3 & 4 & 5 \\
\hline SIZE & 8 & 26 & 88 & 120 & 72 \\
\hline
\end{tabular}

4. Geometry:

Petersen Geometry

Number OF ChAMBERS: 630

DIAMETER: 11

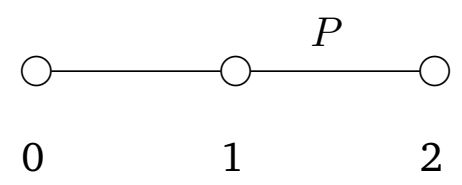

$G_{0} \cong \operatorname{Sym}(5), G_{1} \cong\left(3 \times 2^{2}\right) 2, G_{2} \cong \operatorname{Sym}(4)$,

$G_{01} \cong \operatorname{Dih}(12), G_{02} \cong \operatorname{Dih}(8) \cong G_{12}$,

$B \cong 2^{2}$ (see [8, p. 945]).

\begin{tabular}{|c|c|c|c|c|c|c|c|c|c|c|c|}
\hline DISC & 1 & 2 & 3 & 4 & 5 & 6 & 7 & 8 & 9 & 10 & 11 \\
\hline SIZE & 4 & 8 & 15 & 26 & 42 & 58 & 76 & 104 & 136 & 144 & 16 \\
\hline
\end{tabular}

5. Geometry: Chamber system of type $\tilde{A}_{2}$ $B=1$, $P_{i} \cong 3, i \in\{0,1,2\}$. (See [14, p. 53].)

\begin{tabular}{|c|c|c|c|c|c|c|c|c|c|}
\hline DISC & 1 & 2 & 3 & 4 & 5 & 6 & 7 & 8 & 9 \\
\hline SIZE & 6 & 24 & 72 & 192 & 468 & 851 & 737 & 164 & 5 \\
\hline
\end{tabular}

Remark 2.1. Taking $P_{0}=\langle(123)(456)\rangle, P_{1}=\langle(124)(375)\rangle$ and $P_{2}=$ $\langle(153)(276)\rangle$ (and noting that the chambers are just the elements of Alt(7) and $\left.c_{0}=1\right), D_{9}\left(c_{0}\right)$ looks as follows: 


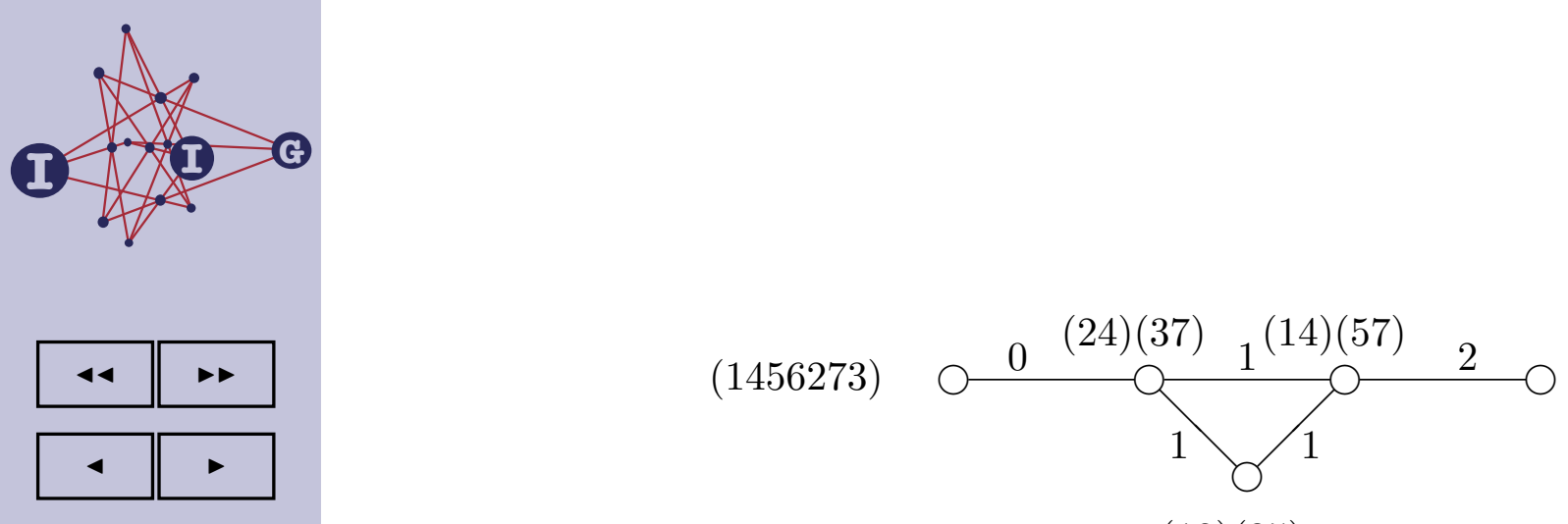

$(1372654)$

(12)(35)

The labels on the edges indicate the $i$-adjacency.

\subsection{Group $G=\operatorname{Sym}(7)$}

GEOMETRY: Number 17 of [4]

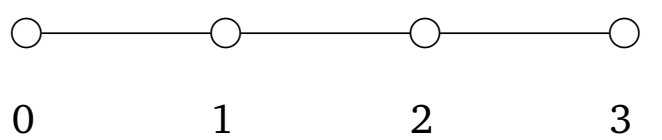

$$
|G|=2^{4} \cdot 3^{2} \cdot 5 \cdot 7=5,040
$$

NuMBER OF CHAMBERS: 840

DIAMETER: 10

The type 0 objects are the elements of a 7-element set $\Omega$ and the objects of type $1,2,3$ are, respectively, all the 2-, 3 - and 4-element subsets of $\Omega$.

$G_{0} \cong \operatorname{Sym}(6), G_{1} \cong 2 \times \operatorname{Sym}(5), G_{2} \cong \operatorname{Sym}(3) \times \operatorname{Sym}(4) \cong G_{3}$,

$G_{01} \cong \operatorname{Sym}(5), G_{02} \cong 2 \times \operatorname{Sym}(4) \cong G_{12}$,

$G_{03} \cong \operatorname{Sym}(3) \times \operatorname{Sym}(3) \cong G_{23}, G_{13} \cong 2^{2} \times \operatorname{Sym}(3)$,

$G_{012} \cong \operatorname{Sym}(4), G_{013} \cong G_{023} \cong G_{123} \cong 2 \times \operatorname{Sym}(3)$,

$B \cong \operatorname{Sym}(3)$.

\begin{tabular}{|c|c|c|c|c|c|c|c|c|c|c|}
\hline DISC & 1 & 2 & 3 & 4 & 5 & 6 & 7 & 8 & 9 & 10 \\
\hline SIZE & 6 & 17 & 39 & 68 & 102 & 136 & 147 & 135 & 108 & 81 \\
\hline
\end{tabular}

\subsection{Group $G=\mathrm{L}_{2}(25)$}

GeOMETRY: Petersen Geometry

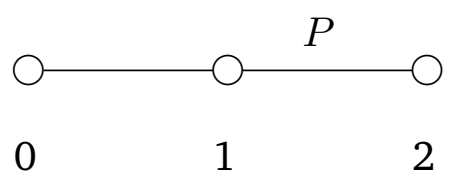

$$
|G|=2^{3} \cdot 3 \cdot 5^{2} \cdot 13=7,800
$$

NuMBER OF CHAMBERS: 1,950

DIAMETER: 18

$G_{0} \cong \operatorname{Sym}(5), G_{1} \cong \operatorname{Dih}(24), G_{2} \cong \operatorname{Sym}(4)$, $G_{01} \cong \operatorname{Dih}(12), G_{01} \cong G_{12} \cong \operatorname{Dih}(8)$,

$B \cong 2^{2}$. (See [8, p. 944].) 


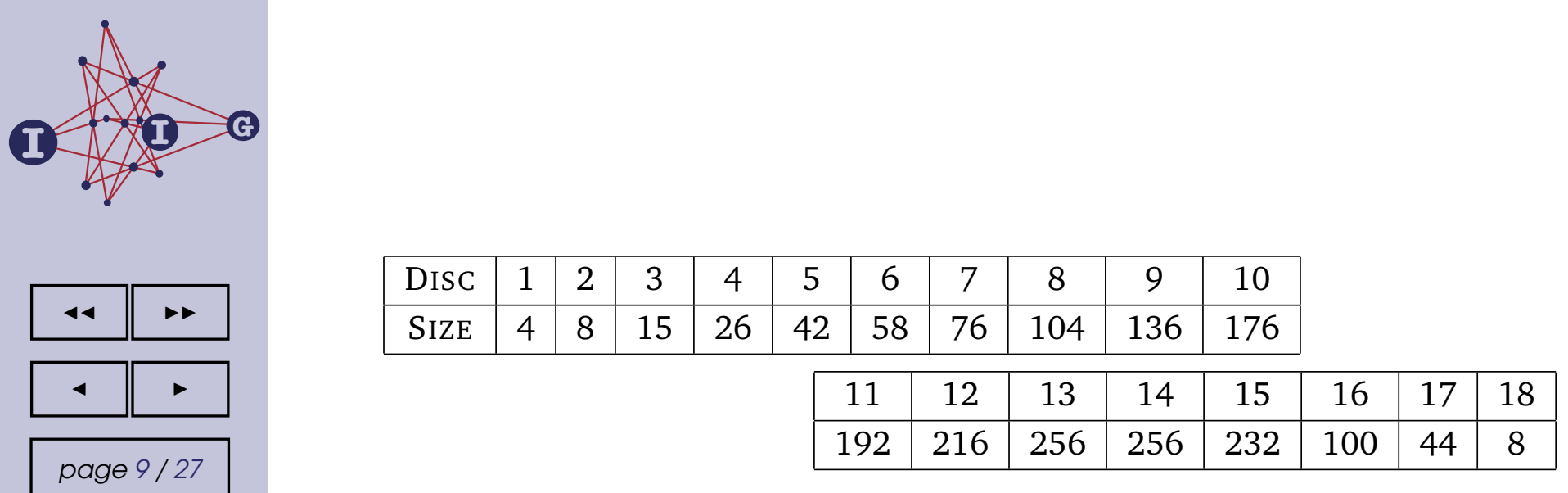

2.6. Group $G=\mathrm{M}_{11}$

$$
|G|=2^{4} \cdot 3^{2} \cdot 5 \cdot 11=7,920
$$

1. Geometry: Number 27 of [4]. Number of Chambers: 2,640

DIAMETER: 11

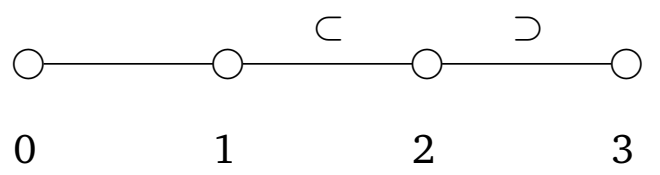

Considering $\mathrm{M}_{11}$ acting 3-transitively on the 12-element set $\Omega$, we may describe the geometry thus. The objects of type 0 and 1 are, respectively all 1- and 2- subsets of $\Omega$; those of type 2 are 3 -element subsets of $\Omega$ of the form $\operatorname{Fix}_{\Omega}(g)$ where $g$ is an element of order 3 in $\mathrm{M}_{11}$ and those of type 3 are one "half" of a total (that is, a 6-element subset of the $6 \mid 6$ partition). Incidence is symmetized containment.

$G_{0} \cong \mathrm{L}_{2}(11), G_{1} \cong \operatorname{Sym}(5), G_{2} \cong 3(\operatorname{Sym}(3) \times 2), G_{3} \cong \operatorname{Alt}(6)$, $G_{01} \cong \operatorname{Alt}(5) \cong G_{03}, G_{02} \cong 2 \times \operatorname{Sym}(3) \cong G_{12}, G_{13} \cong \operatorname{Sym}(4)$, $G_{23} \cong 3^{2}: 2$,

$G_{012} \cong G_{023} \cong G_{123} \cong \operatorname{Sym}(3), G_{013} \cong \operatorname{Alt}(4)$, $B \cong 3$.

\begin{tabular}{|c|c|c|c|c|c|c|c|c|c|c|c|}
\hline DISC & 1 & 2 & 3 & 4 & 5 & 6 & 7 & 8 & 9 & 10 & 11 \\
\hline SIZE & 6 & 19 & 51 & 106 & 204 & 327 & 426 & 534 & 549 & 393 & 24 \\
\hline
\end{tabular}

2. GEOMETRY:

NUMBER OF CHAMBERS: 3,960

DIAMETER: 10

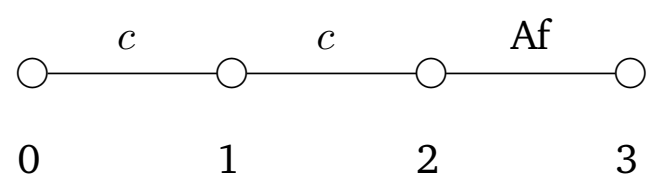

Regarding $\mathrm{M}_{11}$ as the stabilizer of the $S(12,6,5)$ Steiner system on a 12 element set $\Omega$ and an element $\alpha$ of $\Omega$, the geometry may be described 

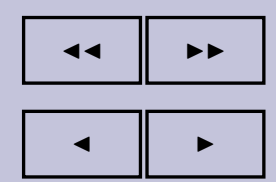

page $10 / 27$

go back

full screen

close

quit

as follows. The objects of type 0,1,2 are, respectively, all 1-, 2- and 3element subsets of $\Omega \backslash\{\alpha\}$ and those of type 3 all the hexads of $S(12,6,5)$ containing $\alpha$. See [13, pp. 72 and 94].

$G_{0} \cong \mathrm{M}_{10}, G_{1} \cong \mathrm{M}_{9}: 2, G_{2} \cong 2 \cdot \operatorname{Sym}(4), G_{3} \cong \operatorname{Sym}(5)$,

$G_{01} \cong 3^{2}: Q_{8}, G_{02} \cong \operatorname{SDih}(16) \cong G_{12}, G_{03} \cong \operatorname{Sym}(4)$,

$G_{13} \cong 2 \times \operatorname{Sym}(3) \cong G_{23}, G_{012} \cong Q_{8}, G_{013} \cong \operatorname{Sym}(3), G_{023} \cong 2^{2} \cong G_{123}$, $B \cong 2$.

(SDih $(n)$ denotes the semidihedral group of order $n$.)

\begin{tabular}{|c|c|c|c|c|c|c|c|c|c|c|}
\hline DISC & 1 & 2 & 3 & 4 & 5 & 6 & 7 & 8 & 9 & 10 \\
\hline SIZE & 7 & 26 & 73 & 155 & 300 & 494 & 636 & 756 & 864 & 648 \\
\hline
\end{tabular}

3. Geometry: Petersen Geometry

Number OF CHAMBERS: 3,960

DIAMETER: 15

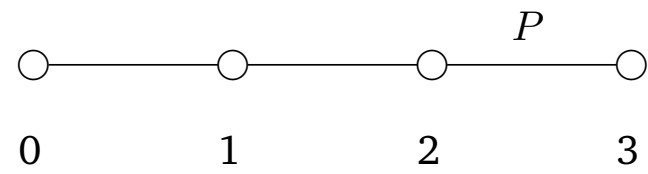

Again starting with $\mathrm{M}_{11}$ acting 3-transitively on a 12-element set $\Omega$, we take as our objects of type $0,1,2$ to be, respectively, all 1-, 2- and 3- subset of $\Omega$ and objects of type 3 to be 4 subsets of the form $\operatorname{Fix}_{\Omega}(g)$ where $g$ is an involution of $\mathrm{M}_{11}$. Incidence being symmeterized inclusion.

$G_{0} \cong \mathrm{L}_{2}(11), G_{1} \cong \operatorname{Sym}(5), G_{2} \cong 3(\operatorname{Sym}(3) \times 2)$,

$G_{3} \cong 2 \cdot \operatorname{Sym}(4), G_{01} \cong \operatorname{Alt}(5), G_{02} \cong G_{03} \cong G_{12} \cong G_{23} \cong 2 \times \operatorname{Sym}(3)$,

$G_{13} \cong \operatorname{Dih}(8), G_{012} \cong \operatorname{Sym}(3), G_{013} \cong G_{023} \cong G_{123} \cong 2^{2}, B \cong 2$.

(See [8, p. 954].)

\begin{tabular}{|c|c|c|c|c|c|c|c|c|c|c|}
\hline DISC & 1 & 2 & 3 & 4 & 5 & 6 & 7 & 8 & 9 & 10 \\
\hline SIZE & 5 & 13 & 28 & 55 & 101 & 171 & 278 & 406 & 516 & 578 \\
\hline
\end{tabular}

\begin{tabular}{|c|c|c|c|c|}
\hline 11 & 12 & 13 & 14 & 15 \\
\hline 612 & 590 & 446 & 144 & 16 \\
\hline
\end{tabular}




\subsection{Group $G=A_{8}$}

GEOMETRY:

$$
|G|=2^{6} \cdot 3^{2} \cdot 5 \cdot 7=20,160
$$

NUMBER OF CHAMBERS: 2,520

DIAMETER: 8

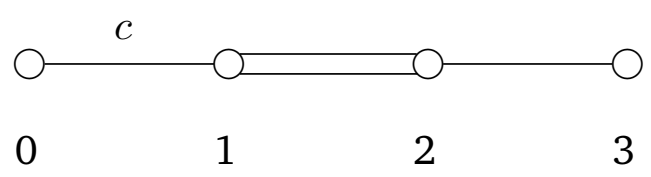

full screen

close

quit

Let $\Omega$ be an 8 -element set. Then the objects of type $0,1,2$ are, respectively the elements, duads and $4^{2}$ partitions of $\Omega$. The $354^{2}$ partitions of $\Omega$ may be identified with the lines of projective 3-space (see [13, Proposition 1]). Objects of type 3 are the points of the projective 3 -space which may be identified with a set of seven $2^{4}$ partitions of $\Omega$ (there are $105=7 \times 152^{4}$ partitions of $\Omega$ ). These seven $2^{4}$ partitions may also be viewed as the non-identity elements of $O_{2}\left(G_{3}\right)$. For the definition of incidence and more details see [12, Section 3].

$G_{0} \cong \operatorname{Alt}(7), G_{1} \cong \operatorname{Sym}(6), G_{2} \cong(\operatorname{Alt}(4) \times \operatorname{Alt}(4)): 2^{2}$,

$G_{3} \cong 2^{3}: \mathrm{L}_{3}(2)$,

$G_{01} \cong \operatorname{Alt}(6), G_{02} \cong(3 \times \operatorname{Alt}(4)): 2, G_{03} \cong \mathrm{L}_{3}(2)$,

$G_{12} \cong 2^{3}: \operatorname{Sym}(3), G_{23} \cong 2^{3}: \operatorname{Sym}(4), G_{13} \cong \operatorname{Sym}(4) \times 2$,

$G_{012} \cong G_{013} \cong G_{023} \cong \operatorname{Sym}(4), G_{123} \cong \operatorname{Dih}(8) \times 2$,

$B \cong \operatorname{Dih}(8)$.

\begin{tabular}{|c|c|c|c|c|c|c|c|c|}
\hline DISC & 1 & 2 & 3 & 4 & 5 & 6 & 7 & 8 \\
\hline SIZE & 7 & 28 & 92 & 256 & 488 & 720 & 744 & 184 \\
\hline
\end{tabular}

\subsection{Group $G=\mathrm{U}_{4}(2)$}

GEOMETRY: Example 6 in [11, Table 4]
$|G|=2^{6} \cdot 3^{4} \cdot 5=25,920$

Number of CHAMBERS: 25,920

DIAMETER: 12

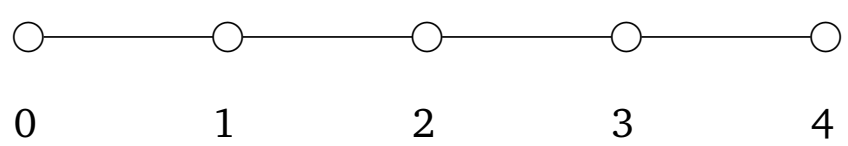

For each $i \in I, G_{J} \cong 3$ for $J=I \backslash\{i\}$ and $B=1$. (See [11] for the other stabilizers.) 

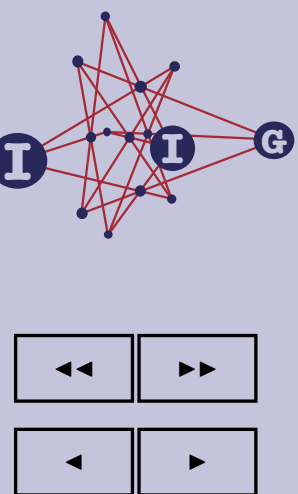

page $12 / 27$

\begin{tabular}{|c|c|c|c|c|c|c|c|c|c|c|}
\hline DISC & 1 & 2 & 3 & 4 & 5 & 6 & 7 & 8 & 9 & 10 \\
\hline SIZE & 10 & 60 & 260 & 855 & 2190 & 4510 & 6930 & 6542 & 3325 & 1150 \\
\hline
\end{tabular}

go back

full screen

close

1. GeOMETRY: Number 5 of [4]

$$
|G|=2^{6} \cdot 3^{3} \cdot 5 \cdot 11=95,040
$$

\subsection{Group $G=\mathrm{M}_{12}$}

\begin{tabular}{|c|c|}
\hline 11 & 12 \\
\hline 85 & 2 \\
\hline
\end{tabular}

\section{quit}

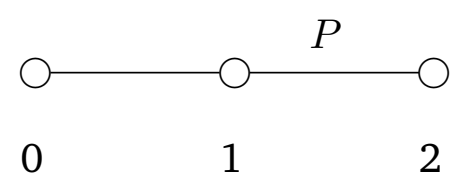

Number OF CHAMBers: 1,320

DIAMETER: 6

With $G$ acting (5-transitively) on the 12 -element set $\Omega$, we take all 1-, 2 and 3-sets of $\Omega$ to be the objects of type $0,1,2$ of the geometry respectively.

$G_{0} \cong \mathrm{M}_{11}, G_{1} \cong \mathrm{M}_{10}: 2, G_{2} \cong 3^{2}: 2 \operatorname{Sym}(4)$,

$G_{01} \cong \mathrm{M}_{10}, G_{02} \cong 3^{2}: \operatorname{SDih}(16) \cong G_{12}$, $B \cong 3^{2}: Q_{8}$.

\begin{tabular}{|c|c|c|c|c|c|c|}
\hline DISC & 1 & 2 & 3 & 4 & 5 & 6 \\
\hline SIZE & 11 & 29 & 118 & 189 & 243 & 729 \\
\hline
\end{tabular}

2. GEOMETRY:

NUMBER OF CHAMBERS: 47,520

DIAMETER: 15

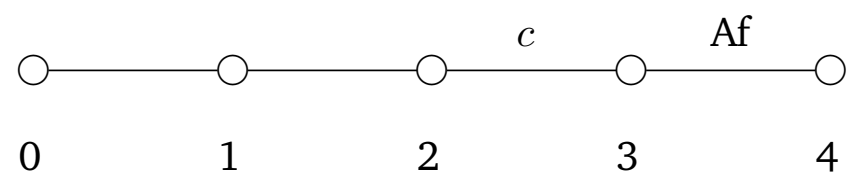

With $\Omega$ a 12-element set, the objects of type $0,1,2,3$ and 4 are, respectively 1 -, 2-, 3-, 4-subsets of $\Omega$ and the hexads of the Steiner system $S(12,6,5)$. See [13, pp. 72 and 94].

$G_{0} \cong \mathrm{M}_{11}, G_{1} \cong \operatorname{Alt}(6) \cdot 2^{2} \cong G_{4}, G_{2} \cong 3^{2}: 2 \operatorname{Sym}(4), G_{3} \cong 2_{+}^{1+4}: \operatorname{Sym}(3)$, $G_{0123} \cong Q_{8}, G_{0124} \cong \operatorname{Sym}(3), G_{0134} \cong G_{0234} \cong G_{1234} \cong 2^{2}, B \cong 2$. 

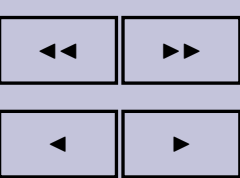

page $13 / 27$

go back

full screen

close

quit

\begin{tabular}{|c|c|c|c|c|c|c|c|c|c|c|}
\hline DISC & 1 & 2 & 3 & 4 & 5 & 6 & 7 & 8 & 9 & 10 \\
\hline SIZE & 8 & 34 & 107 & 263 & 574 & 1116 & 1887 & 2934 & 4280 & 5692 \\
\hline
\end{tabular}

\begin{tabular}{|c|c|c|c|c|}
\hline 11 & 12 & 13 & 14 & 15 \\
\hline 6504 & 6840 & 6912 & 6480 & 3888 \\
\hline
\end{tabular}

2.10. Group $G=\mathrm{U}_{3}(5)$

$$
|G|=2^{4} \cdot 3^{2} \cdot 5^{3} \cdot 7=126,000
$$

1. GEOMETRY:

Number OF CHAMBERS: 15,750

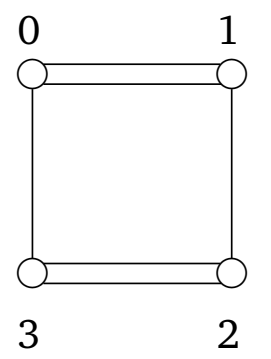

DIAMETER: 10

See [7] and [12].

$G_{0} \cong G_{1} \cong G_{2} \cong G_{3} \cong \operatorname{Alt}(7), G_{01} \cong G_{23} \cong \operatorname{Alt}(6)$,

$G_{02} \cong G_{13} \cong(3 \times$ Alt $(4)): 2, G_{03} \cong G_{12} \cong \mathrm{L}_{3}(2)$,

$G_{012} \cong G_{013} \cong G_{023} \cong G_{123} \cong \operatorname{Sym}(4), B \cong \operatorname{Dih}(8)$.

\begin{tabular}{|c|c|c|c|c|c|c|c|c|c|c|}
\hline DISC & 1 & 2 & 3 & 4 & 5 & 6 & 7 & 8 & 9 & 10 \\
\hline SIZE & 8 & 40 & 176 & 704 & 2080 & 4748 & 5680 & 2060 & 252 & 1 \\
\hline
\end{tabular}

2. GeOMETRY:

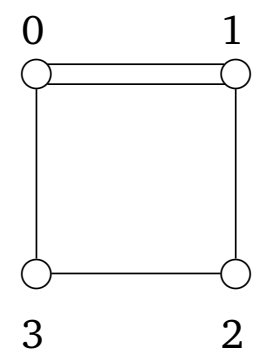

NUMBER OF CHAMBERS: 15,750

DIAMETER: 10

See [7] and [19].

$G_{0} \cong G_{1} \cong G_{2} \cong G_{3} \cong \operatorname{Alt}(7), G_{01} \cong G_{03} \cong \mathrm{L}_{3}(2)$,

$G_{02} \cong G_{13} \cong(3 \times \operatorname{Alt}(4)): 2, G_{12} \cong \mathrm{L}_{3}(2), G_{23} \cong \mathrm{Alt}(6)$,

$G_{012} \cong G_{013} \cong G_{023} \cong G_{123} \cong \operatorname{Sym}(4), B \cong \operatorname{Dih}(8)$. 

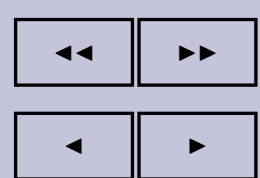

go back

full screen

close

quit

\begin{tabular}{|c|c|c|c|c|c|c|c|c|c|c|}
\hline DISC & 1 & 2 & 3 & 4 & 5 & 6 & 7 & 8 & 9 & 10 \\
\hline SIZE & 8 & 40 & 168 & 624 & 1840 & 4628 & 6776 & 1620 & 44 & 1 \\
\hline
\end{tabular}

\subsection{Group $G=\mathrm{J}_{1}$}

GEOMETRY:

Number 28 of [4]

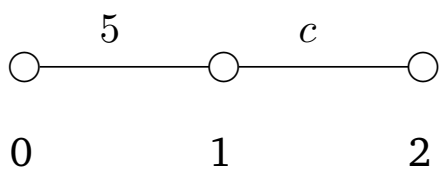

$|G|=2^{3} \cdot 3 \cdot 5 \cdot 7 \cdot 11 \cdot 19=175,500$

Number OF ChAMBERS: 29,260

DIAMETER: 12

$G_{0} \cong \mathrm{L}_{2}(11), G_{1} \cong 2 \times \operatorname{Alt}(5), G_{2} \cong \operatorname{Sym}(3) \times \operatorname{Dih}(10)$,

$G_{01} \cong \operatorname{Alt}(5), G_{02} \cong G_{12} \cong 2 \times \operatorname{Sym}(3)$,

$B \cong \operatorname{Sym}(3)$.

\begin{tabular}{|c|c|c|c|c|c|c|c|c|c|c|}
\hline DISC & 1 & 2 & 3 & 4 & 5 & 6 & 7 & 8 & 9 & 10 \\
\hline SIZE & 11 & 29 & 119 & 209 & 379 & 1260 & 2124 & 3960 & 9402 & 8196 \\
\hline
\end{tabular}

\begin{tabular}{|c|c|}
\hline 11 & 12 \\
\hline 3102 & 468 \\
\hline
\end{tabular}

\subsection{Group $G=\operatorname{Alt}(9)$}

GEOMETRY:

Petersen Geometry

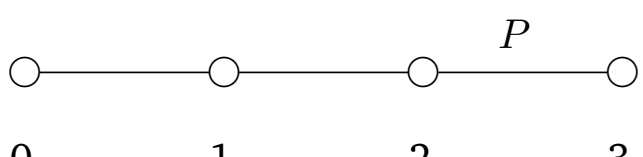

See [8].

$G_{0} \cong \operatorname{Sym}(7), G_{1} \cong 2 . \operatorname{Sym}(5), G_{2} \cong \operatorname{Sym}(4) \times \operatorname{Sym}(3), G_{3} \cong 2^{3} \operatorname{Sym}(4)$,

$\left|G_{012}\right|=2^{3} 3,\left|G_{013}\right|=\left|G_{023}\right|=\left|G_{123}\right|=2^{4}$,

$|B|=2^{3}$.

0

1

3
$|G|=2^{6} \cdot 3^{4} \cdot 5 \cdot 7=1,811,440$

Number of ChAMBERs: 22,680

DIAMETER: 18 


\subsection{Group $G=$ Aut $\mathrm{M}_{22}$}

GEOMETRY:

Petersen Geometry

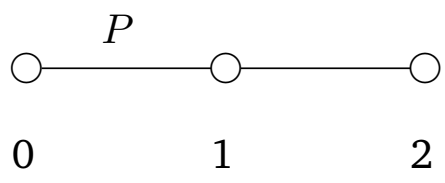

$|G|=2^{8} \cdot 3^{2} \cdot 5 \cdot 7 \cdot 11=887,040$

Number OF ChAMBers: 6,930

DIAMETER: 13 go back

full screen

close

quit

It is convenient to describe this geometry by beginning with a 24-element set $\Omega$, assumed to be equipped with the Steiner system $S(24,8,5)$. Now fix a duad $D$ (2-element subset) of $\Omega$. Then $\operatorname{Stab}_{\mathrm{M}_{24}} D \cong$ Aut $\mathrm{M}_{22}$ and objects of type $0,1,2$ of the geometry are, respectively, all octads in $\Omega \backslash D$, all trios which have $D$ contained in one of its octads and all sextets which have $D$ contained in one of its tetrads. Incidence being given by compatibility of partitions (see [9]).

$G_{0} \cong\left(2^{3}: \mathrm{L}_{3}(2)\right) \times 2, G_{1} \cong 2^{1+4}\left(2^{2} \times \operatorname{Sym}(3)\right.$,

$G_{2} \cong 2^{5}: \operatorname{Sym}(5), G_{01} \cong G_{02} \cong\left(2^{3}: \operatorname{Sym}(4)\right) \times 2$, $G_{12} \cong 2^{5}: \operatorname{Dih}(8), B \cong\left(2^{3}: \operatorname{Dih}(8)\right) \times 2$.

\begin{tabular}{|c|c|c|c|c|c|c|c|c|c|c|}
\hline DISC & 1 & 2 & 3 & 4 & 5 & 6 & 7 & 8 & 9 & 10 \\
\hline SIZE & 5 & 14 & 30 & 56 & 112 & 200 & 320 & 512 & 800 & 1248 \\
\hline
\end{tabular}

\begin{tabular}{|c|c|c|}
\hline 11 & 12 & 13 \\
\hline 1808 & 1696 & 128 \\
\hline
\end{tabular}

Remark 2.2. $D_{13}\left(c_{0}\right)$ is a $B$-orbit.

\subsection{Group $G=3 \mathrm{M}_{22}$}

GEOMETRY: $|G|=2^{7} \cdot 3^{3} \cdot 5 \cdot 7 \cdot 11=1,330,560$

NuMBER OF CHAMBERS: 10,395

DIAMETER: 8

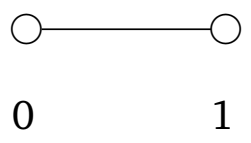

This is a 3 -fold cover of the minimal parabolic geometry for $M_{22}$ (see section 2.13).

$G_{0} \cong 2^{4}: \operatorname{Sym}(5), G_{1} \cong 2^{6} \operatorname{Sym}(3), B \cong 2^{4}: \operatorname{Dih}(8)$. 


\begin{tabular}{|c|c|c|c|c|c|c|c|c|}
\hline DISC & 1 & 2 & 3 & 4 & 5 & 6 & 7 & 8 \\
\hline SIZE & 16 & 56 & 432 & 1056 & 3632 & 4304 & 872 & 26 \\
\hline
\end{tabular}

2.16. Group $G=3 \mathrm{M}_{22} 2$

page $17 / 27$

go back

full screen

close

quit
GEOMETRY:

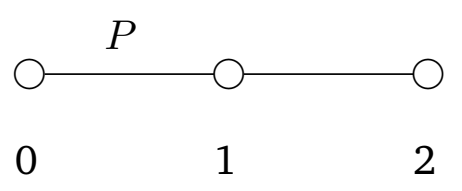

$|G|=2^{8} \cdot 3^{3} \cdot 5 \cdot 7 \cdot 11=2,661,120$

Number of Chambers: 20,790

DIAMETER: 24

A 3-fold cover of the Petersen geometry given in section 2.14 (see also [9]).

$G_{0} \cong\left(2^{3}: \mathrm{L}_{3}(2)\right) \times 2, G_{1} \cong 2^{1+4}\left(2^{2} \times \operatorname{Sym}(3)\right)$,

$G_{2} \cong 2^{5}: \operatorname{Sym}(5), G_{01} \cong G_{02} \cong\left(2^{3}: \operatorname{Sym}(4)\right) \times 2$,

$G_{12} \cong 2^{5}: \operatorname{Dih}(8), B \cong\left(2^{3}: \operatorname{Dih}(8)\right) \times 2$.

\begin{tabular}{|c|c|c|c|c|c|c|c|c|c|c|}
\hline DISC & 1 & 2 & 3 & 4 & 5 & 6 & 7 & 8 & 9 & 10 \\
\hline SIZE & 5 & 14 & 30 & 56 & 112 & 200 & 320 & 512 & 800 & 1248 \\
\hline
\end{tabular}

\begin{tabular}{|c|c|c|c|c|c|c|c|c|c|}
\hline 11 & 12 & 13 & 14 & 15 & 16 & 17 & 18 & 19 & 20 \\
\hline 1808 & 2368 & 3008 & 3968 & 3456 & 1216 & 736 & 464 & 248 & 120 \\
\hline
\end{tabular}

Remark 2.3. Note that the sizes here of $D_{i}\left(c_{0}\right)$ for $1 \leq i \leq 11$ coincide with those in section 2.14 .

\subsection{Group $G=G_{2}(3)$}

$$
|G|=2^{6} \cdot 3^{6} \cdot 7 \cdot 13=4,245,696
$$

1. GeOMETRY:

Number OF CHAMBERS: 66,339

DIAMETER: 13

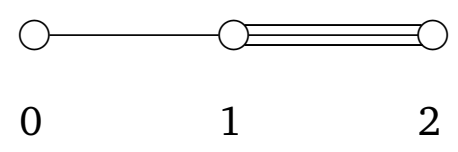

See [1]. 

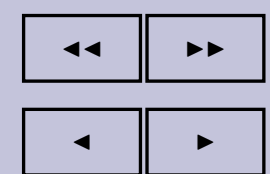

page $18 / 27$

go back

full screen

close

quit

$G_{0} \cong 2^{3}: \mathrm{L}_{3}(2), G_{1} \cong 2_{+}^{1+4}: 3^{2} \cdot 2$,

$G_{3} \cong G_{2}(2), G_{01} \cong G_{02} \cong G_{12} \cong 2^{5} \cdot \operatorname{Sym}(3)$,

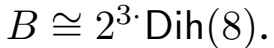

\begin{tabular}{|c|c|c|c|c|c|c|c|c|c|c|}
\hline DISC & 1 & 2 & 3 & 4 & 5 & 6 & 7 & 8 & 9 & 10 \\
\hline SIZE & 6 & 20 & 56 & 144 & 384 & 960 & 2176 & 4864 & 10368 & 10972 \\
\hline
\end{tabular}

\begin{tabular}{|c|c|c|}
\hline 11 & 12 & 13 \\
\hline 21248 & 6976 & 64 \\
\hline
\end{tabular}

2. GEOMETRY:

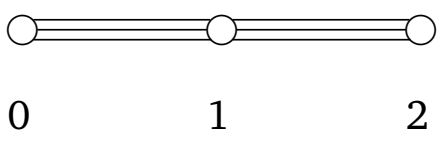

See [1].

$G_{0} \cong G_{2}(2) \cong G_{2}, G_{1} \cong 2_{+}^{1+4}: 3^{2} \cdot 2$,

$G_{01} \cong G_{02} \cong G_{12} \cong 2^{5} \cdot \operatorname{Sym}(3)$,

$B \cong 2^{3 \cdot} \operatorname{Dih}(8)$.

\begin{tabular}{|c|c|c|c|c|c|c|c|c|c|c|}
\hline DISC & 1 & 2 & 3 & 4 & 5 & 6 & 7 & 8 & 9 & 10 \\
\hline SIZE & 6 & 20 & 64 & 208 & 600 & 1728 & 4640 & 10368 & 17920 & 20416 \\
\hline
\end{tabular}

2.18. Group $G=\mathrm{U}_{4}(3) 2$

GEOMETRY:

$|G|=2^{8} \cdot 3^{6} \cdot 5 \cdot 7=6,531,840$

Number OF CHAMBERS: 25,515

DIAMETER: 10

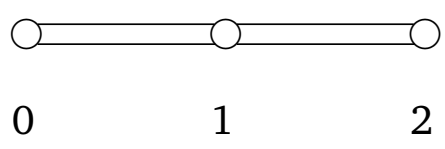

This geometry is an example of a GAB — see [10, Section 3] for details.

$G_{0} \cong 2^{4} \cdot \operatorname{Sym}(6), G_{1} \cong\left[2^{6}.\right] \cdot(\operatorname{Sym}(3) \times \operatorname{Sym}(3)), G_{2} \cong 2^{5}$.Alt $(6)$,

$G_{01} \cong G_{02} \cong G_{12} \cong\left[2^{6}\right]$.Sym(3),

$B \cong 2^{4} \cdot(\operatorname{Dih}(8) \times 2)$. 


\begin{tabular}{|c|c|c|c|c|c|c|c|c|c|c|}
\hline DISC & 1 & 2 & 3 & 4 & 5 & 6 & 7 & 8 & 9 & 10 \\
\hline SIZE & 6 & 20 & 64 & 176 & 416 & 1024 & 2432 & 5120 & 9088 & 7168 \\
\hline
\end{tabular}

2.19. Group $G=\mathrm{U}_{5}(2)$

page $19 / 27$

go back

full screen

close

quit
GEOMETRY:

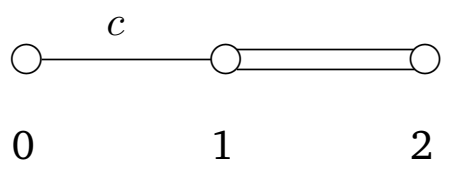

$|G|=2^{10} \cdot 3^{5} \cdot 5 \cdot 11=13,685,760$

Number of ChAMBERS: 28,160

DIAMETER: 11

Number 20 of [4] (with $n=5$ ).

$G_{0} \cong 3 \times \mathrm{U}_{4}(2), G_{1} \cong \operatorname{Sym}(3) \times\left(3^{1+2}: \mathrm{SL}_{2}(3)\right), G_{2} \cong 3^{4} \cdot \operatorname{Sym}(5)$,

$G_{01} \cong 3^{4} \cdot \mathrm{SL}_{2}(3), G_{02} \cong 3^{4} \cdot \operatorname{Sym}(4), G_{12} \cong 3^{5}: 2^{2}$, $B=3^{5}: 2$.

\begin{tabular}{|c|c|c|c|c|c|c|c|c|c|c|c|}
\hline DISC & 1 & 2 & 3 & 4 & 5 & 6 & 7 & 8 & 9 & 10 & 11 \\
\hline SIZE & 7 & 27 & 99 & 270 & 594 & 1431 & 3051 & 5427 & 8019 & 8262 & 972 \\
\hline
\end{tabular}

2.20. Group $G=\mathrm{M}_{23}$

$$
|G|=2^{7} \cdot 3^{2} \cdot 5 \cdot 7 \cdot 11 \cdot 23=10,200,960
$$

1. GeOMETRY:

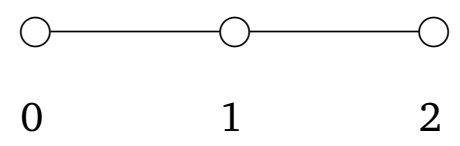

Minimal parabolic "1-geometry" for $\mathrm{M}_{23}$ (see [16]).

$G_{0} \cong \mathrm{M}_{22}, \quad G_{1} \cong 2^{4}(3 \times \operatorname{Alt}(5)) 2, \quad G_{2} \cong 2^{4} \mathrm{~L}_{3}(2)$,

$G_{01} \cong 2^{4} \operatorname{Sym}(5), \quad G_{02} \cong 2^{4} \operatorname{Sym}(4) \cong G_{12}$,

$B \cong 2^{4} \operatorname{Dih}(8)$.

\begin{tabular}{|c|c|c|c|c|c|c|c|}
\hline DISC & 1 & 2 & 3 & 4 & 5 & 6 & 7 \\
\hline SIZE & 18 & 92 & 664 & 3104 & 10728 & 36032 & 29056 \\
\hline
\end{tabular}

Remark 2.4. $\mathrm{M}_{23}$ has two (non-isomorphic) minimal parabolic geometries which are locally isomorphic (meaning all their residues are isomorphic). Globally they differ with the choice of an $L_{3}(2)$-conjugacy class 


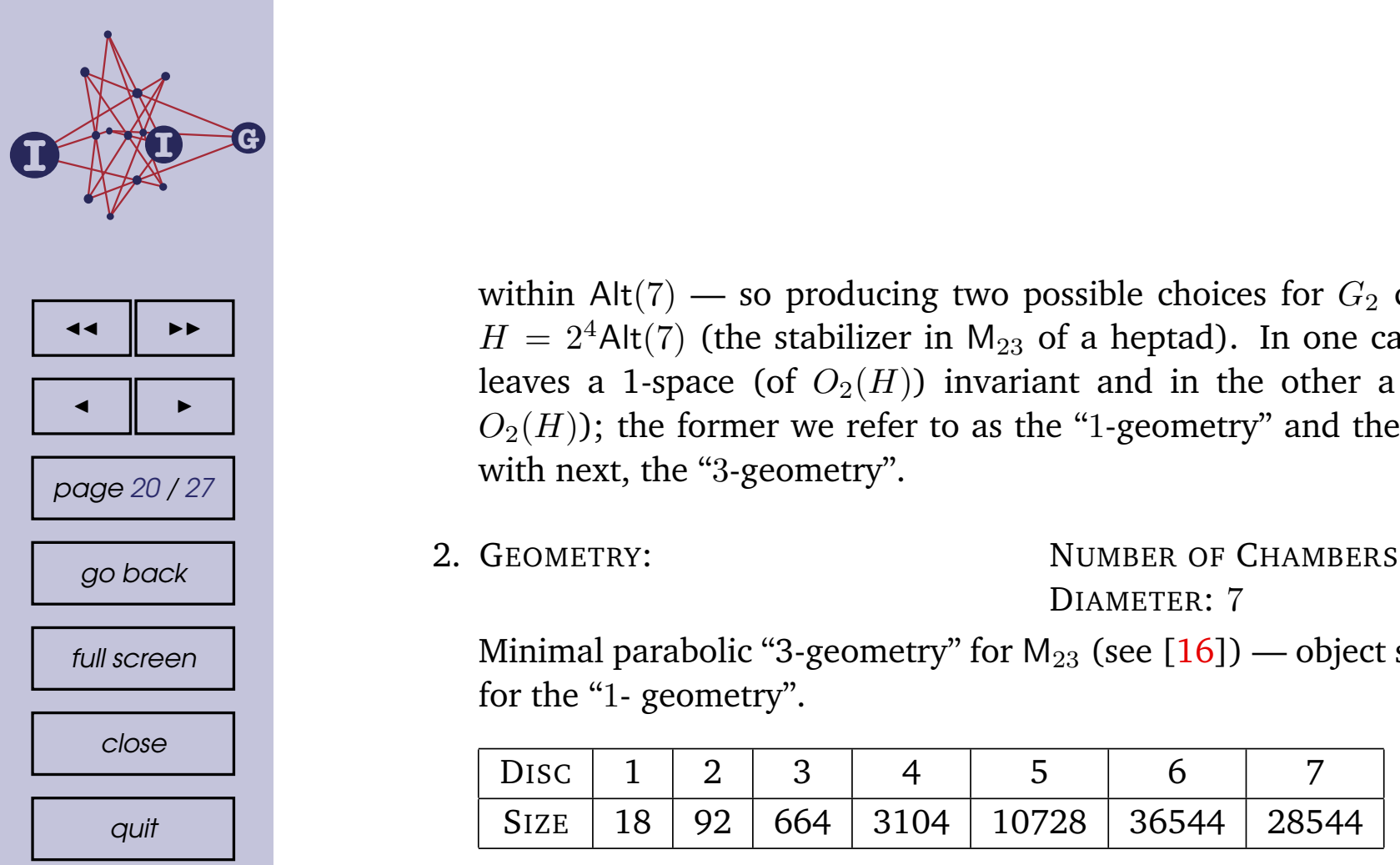

within Alt $(7)$ - so producing two possible choices for $G_{2}$ contained in $H=2^{4} \mathrm{Alt}(7)$ (the stabilizer in $\mathrm{M}_{23}$ of a heptad). In one case the $\mathrm{L}_{3}(2)$ leaves a 1-space (of $\mathrm{O}_{2}(H)$ ) invariant and in the other a 3-space (of $\mathrm{O}_{2}(\mathrm{H})$ ); the former we refer to as the "1-geometry" and the latter, dealt with next, the " 3 -geometry".

2. Geometry:

NuMBeR OF CHAMBERS: 79,695

DIAMETER: 7

Minimal parabolic "3-geometry" for $\mathrm{M}_{23}$ (see [16]) — object stabilizers as for the "1- geometry".

\begin{tabular}{|c|c|c|c|c|c|c|c|}
\hline DISC & 1 & 2 & 3 & 4 & 5 & 6 & 7 \\
\hline SIZE & 18 & 92 & 664 & 3104 & 10728 & 36544 & 28544 \\
\hline
\end{tabular}

Remark 2.5. The disc sizes of the 1-geometry and the 3-geometry differ only in discs 6 and 7 - the 1-geometry has 512 fewer chambers in the sixth disc and 512 more in the seventh disc (than the 3-geometry).

3. GEOMETRY:

Number OF CHAMBERS: 53,130

DIAMETER: 10

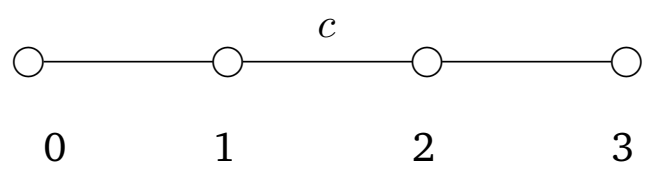

Number 44 of [4].

$G_{0} \cong \mathrm{M}_{22}, G_{1} \cong: \mathrm{L}_{3}(4) 2, G_{2} \cong 2^{4}:(3 \times \operatorname{Alt}(5)): 2$,

$G_{3} \cong 2^{4}: \operatorname{Alt}(7), G_{012} \cong G_{013} \cong 2^{4} \operatorname{Alt}(5), G_{023} \cong G_{123} \cong 2^{4} \operatorname{Sym}(4)$, $B \cong 2^{4} \mathrm{Alt}(4)$.

\begin{tabular}{|c|c|c|c|c|c|c|c|c|c|c|}
\hline DISC & 1 & 2 & 3 & 4 & 5 & 6 & 7 & 8 & 9 & 10 \\
\hline SIZE & 10 & 54 & 201 & 560 & 1552 & 3392 & 5376 & 9216 & 16384 & 16384 \\
\hline
\end{tabular}

4. Geometry:

Petersen Geometry

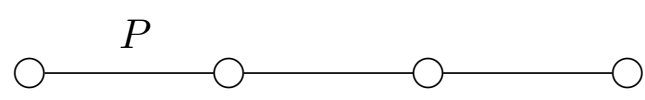

0

$2 \quad 3$

Number OF ChAmbers: 159,390

DIAMETER: 14 

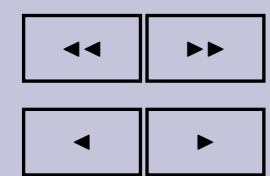

page $21 / 27$

go back

full screen

close

quit
$G_{0} \cong \operatorname{Alt}(8), G_{1} \cong 2^{3}\left(\mathrm{~L}_{3}(2) \times 2\right), G_{2} \cong 2^{4}(3 \times \operatorname{Alt}(5)) 2$,

$G_{3} \cong \mathrm{M}_{22}$,

$G_{012} \cong G_{013} \cong G_{023} \cong 2^{3}: \operatorname{Sym}(4), G_{123} \cong 2^{4}: \operatorname{Dih}(8)$, $B \cong 2^{3}: \operatorname{Dih}(8)$.

\begin{tabular}{|c|c|c|c|c|c|c|c|c|c|c|}
\hline DISC & 1 & 2 & 3 & 4 & 5 & 6 & 7 & 8 & 9 & 10 \\
\hline SIZE & 7 & 28 & 86 & 220 & 512 & 1128 & 2432 & 5152 & 10528 & 21024 \\
\hline
\end{tabular}

\begin{tabular}{|c|c|c|c|}
\hline 11 & 12 & 13 & 14 \\
\hline 38528 & 51840 & 26304 & 1600 \\
\hline
\end{tabular}

\subsection{Group $G=3 U_{4}(3) 2$}

GEOMETRY:

$$
|G|=2^{8} \cdot 3^{7} \cdot 5 \cdot 7=19,595,520
$$

NuMBER OF CHAMBERS: 76,545

DIAMETER: 11

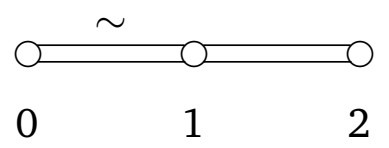

This geometry is a triple cover of the geometry in section 2.18.

$G_{0} \cong 2^{4}: \operatorname{Sym}(6), G_{1} \cong\left[2^{6}\right](\operatorname{Sym}(3) \times \operatorname{Sym}(3)), G_{2} \cong 2^{5} .3 \operatorname{Alt}(6)$, $G_{01} \cong G_{02} \cong G_{12} \cong\left[2^{7}\right] \operatorname{Sym}(3), B \cong 2^{4}(\operatorname{Dih}(8) \times 2)$.

\begin{tabular}{|c|c|c|c|c|c|c|c|c|c|c|}
\hline DISC & 1 & 2 & 3 & 4 & 5 & 6 & 7 & 8 & 9 & 10 \\
\hline SIZE & 6 & 20 & 64 & 192 & 528 & 1424 & 3848 & 9658 & 19812 & 27680 \\
\hline
\end{tabular}

\begin{tabular}{|c|}
\hline 11 \\
\hline 13312 \\
\hline
\end{tabular}

2.22. Group $G=\mathrm{M}_{24}$

$$
|G|=2^{10} \cdot 3^{3} \cdot 5 \cdot 7 \cdot 11 \cdot 23=244,823,040
$$

1. GEOMETRY:

Maximal 2-local

geometry (see [15])

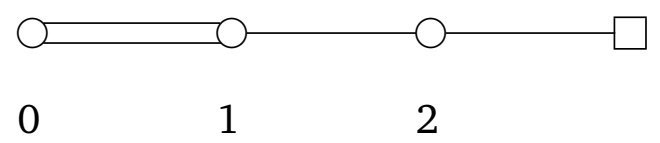

NUMBER OF CHAMBERS: 79,695

DIAMETER: 10 

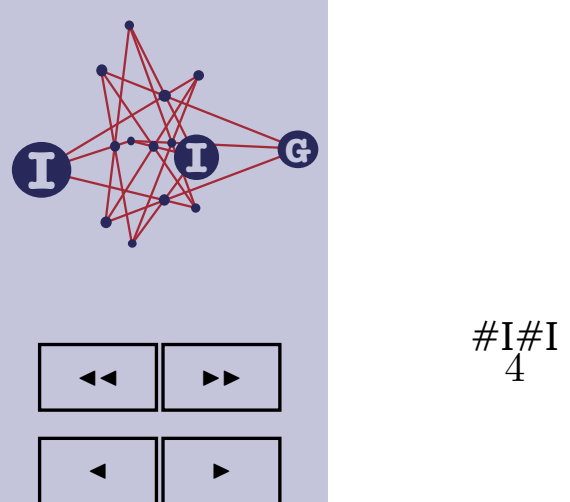

page $24 / 27$

go back

full screen

close

quit

$6 \quad 4$

$($ (2)-10) fano.eps\# \# \#I and 1 [c]0.3 (2,-10) fano.ep\$I \#I are two chambers which have the same point and line - they are 3 -adjacent.

panelbackground

Remark 3.1. Using the action of elements of $B$, from Table ?? we may obtain the edge set for the induced graph on $D_{5}\left(c_{0}\right)$.

Theorem 3.2. The induced subgraph on $D_{5}\left(c_{0}\right)$ is a connected graph.

Proof. Let $\mathcal{E}$ denote the connected component of $c_{8}$ (see p. ??) in the subgraph $D_{5}\left(c_{0}\right)$. Also put $E=\operatorname{Stab}_{B}(\mathcal{E})$. From ??, $c_{8}$ and $c_{8}(23)(67)$ are 3 -adjacent and so $c_{8}(23)(67) \in \mathcal{E}$. Hence $(23)(67) \in E$. Also, by Table ??, $c_{11}$ is 2 -adjacent to $c_{8}$ and $c_{11}$ is 2 -adjacent to

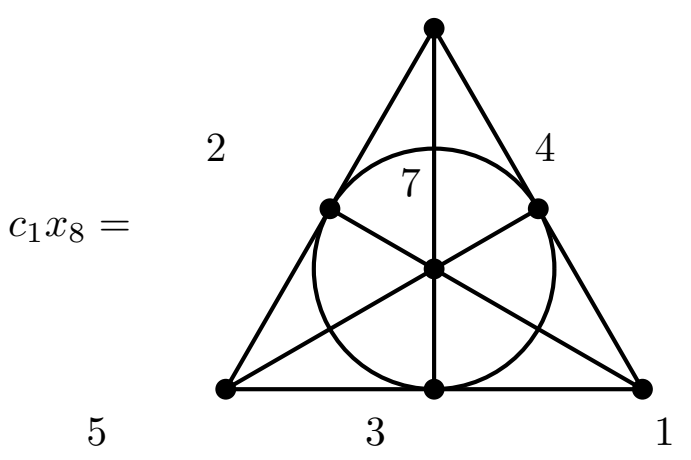

Therefore $c_{1} x_{8} \in \mathcal{E}$. Again from Table ??, $c_{8}$ is 1 -adjacent to 


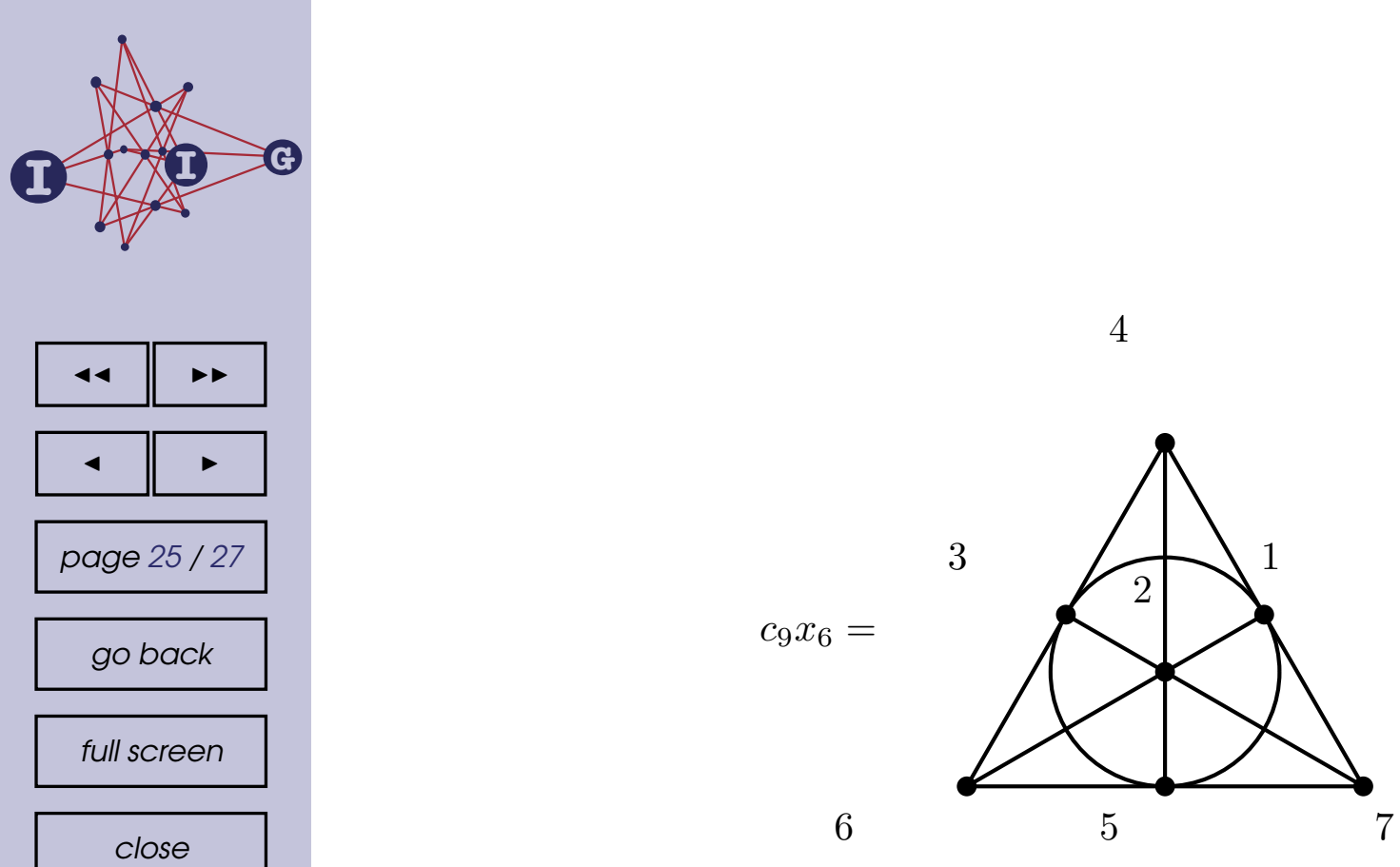

Now $c_{9} x_{6}$ is 2 -adjacent to

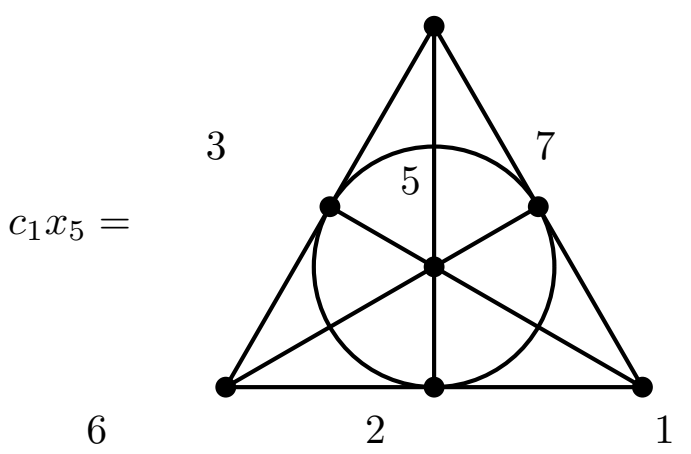

So $c_{1} x_{5} \in \mathcal{E}$. Since $(23)(4657)$ sends $c_{1} x_{5}$ to $c_{1} x_{8},(23)(4657) \in E$. Thus, as $B=\langle(23)(67),(23)(4657)\rangle, E=B$. Inspecting Table ?? we see that there is a path in $D_{5}\left(c_{0}\right)$ from $c_{8}$ to a chamber in each $c_{i}^{B}(i \in\{1, \ldots, 13\})$. Therefore $\mathcal{E}=D_{5}\left(c_{0}\right)$, and this completes the proof.

\section{References}

[1] M. Aschbacher and S. D. Smith, Tits geometries over $G F(2)$ defined by groups over $G F(3)$, Comm. Algebra 11 (1983), 1675-1684.

[2] F. Buekenhout, The geometry of diagrams, Geom. Dedicata 8 (1979), 253-257.

[3] _ Diagrams for geometries and groups, J. Combin. Theory Ser. A, 27 (1979), 121-151. 
[4] _ Diagrams for sporadic groups, in Finite Groups - Coming of Age, J. McKay, ed., Contemp. Math. 45 (1985), pp. 1-32.

[5] J. J. Cannon and C. Playoust, An Introduction to Algebraic Programming with MAGMA, Draft, 1997.

[6] J. H. Conway, R. T. Curtis, S. P. Norton, R. A. Parker and R. A. Wilson, Atlas of Finite Groups. Maximal subgroups and ordinary characters for simple groups, Oxford Univ. Press, Eynsham, 1985.

[7] S. Heiss, Two sporadic geometries related to the Hoffman-Singleton graph, J. Combin. Theory Ser. A 53 (1990), 68-80.

[8] A. A. Ivanov and S. V. Shpectorov, Geometries for sporadic groups related to the Petersen graph I, Comm. Algebra 16 (1988), 925-953.

[9] _ Geometries for sporadic groups related to the Petersen graph II, European J. Combin. 10 (1989), 347-362.

[10] W. M. Kantor, Some Geometries that are Almost Buildings, European J. Combin. 2 (1981), 239-247.

[11] E. A. Komissartschik and S. V. Tsaranov, Construction of finite groups amalgams and geometries. Geometries of the group $\mathrm{U}_{4}(2)$, Comm. Algebra 18 (1990), 1071-1117.

[12] A. Neumaier, Some sporadic geometries related to $P G(3,2)$, Arch. Math., 42 (1984), 89-96.

[13] A. Pasini, Diagram Geometries, Oxford Sci. Publ., Oxford Univ. Press, New York, 1994.

[14] M. A. Ronan, Lectures on Buildings, Persp. Math., Academic Press, Boston, 1989.

[15] M. A. Ronan and S. D. Smith, 2-local geometries for some sporadic groups, in The Santa Cruz Conference on Finite Groups (Univ. California, Santa Cruz, 1979), Proc. Symp. Pure Math. 37 (1980), pp. 283-289.

[16] M. A. Ronan and G. Stroth, Minimal parabolic geometries for the sporadic groups, European J. Combin. 5 (1984), 59-91.

[17] P. Rowley, The Chamber graph of the $\mathrm{M}_{24}$-maximal 2-local geometry, $J$. Comput. Math. 12 (2009), 120-143.

[18] J. Tits, A local approach to buildings. in The Geometric Vein, The Coxeter Festschrift (1981), 519-547. 
[19] M. Wester, Endliche fahnentransitive Tits-Geometrien and ihre universellen Überlagerungen, Mitt. Math. Sem. Giessen 170 (1985), 143 pp.

\section{P. J. Rowley}

School of Mathematics, University of Manchester, Oxford Road, Manchester, M13 9PL, UnITED KINGDOM

e-mail: Peter.J.Rowley@manchester.ac.uk

close

quit go back

full screen 